\title{
A multi-site study of the extreme helium star V2076 Oph
}

\author{
D.J. Wright ${ }^{1}$, P.L. Cottrell ${ }^{1}$, A.E. Lynas-Gray ${ }^{2}$, D. Kilkenny ${ }^{3}$, \\ R. Shobbrook ${ }^{4}$ \\ ${ }^{1}$ Department of Physics \& Astronomy, University of Canterbury, \\ Private Bag 4800, Christchurch, New Zealand \\ ${ }^{2}$ Department of Physics, University of Oxford, Oxford OX13RH, UK \\ ${ }^{3}$ South African Astronomical Observatory, PO Box 9, Observatory \\ 7935, South Africa \\ ${ }^{4}$ School of Physics, University of Sydney, NSW 2006, Australia
}

\begin{abstract}
Results from a three-site photometric and spectroscopic campaign of the hottest known extreme helium star V2076 Oph are presented. V2076 Oph shows complex multiple periods ranging from 0.3 to $3 \mathrm{~d}$. However for the two campaigns in 1982 and 2000 , no definitive single period can be identified in both datasets. Some of the extracted periods appear to be one-day aliases of others. The two most convincing periods are $0.52 \mathrm{~d}(22.28 \mathrm{mHz})$ and $2.75 \mathrm{~d}(4.21 \mathrm{mHz})$. The spectral lines identified in V2076 Oph were He I, HeII, C II (emission), C III (both emission and absorption), CIV and N III. These lines showed profile variations that could not be linked to the photometric variations. Individual line velocities vary by species and ionisation level. The spectral lines vary in phase with an amplitude of $\sim 25 \mathrm{~km} \mathrm{~s}^{-1}$ - the exception is the strong C III emission line at $5696 \AA$ which shows different behaviour and has the lowest observed average velocity. This indicates that this emission line originates in the stellar wind rather than the photosphere.
\end{abstract}

\section{Introduction}

V2076 Oph (HD 160641) was recognised as a hot extreme helium (EHe) star by Bidelman (1952). Aller (1954) showed that carbon was the most abundant element after helium and a non-LTE abundance analysis (Rauch 1996) revealed $99 \%$ He and $1 \% \mathrm{C}$ by number. These abundances have been considered typical for EHe stars since Schönberner \& Wolf (1974) analysed the prototype, HD 124448. Hamman et al. (1982) found pronounced P Cygni profiles in the ultraviolet spectra from which they deduced a mass-loss rate of $\sim 10^{-7} \mathrm{M}_{\odot} \mathrm{yr}^{-1}$. Schönberner (1977) proposed that EHe stars have a $\mathrm{C} / \mathrm{O}$ core and helium burning in a shell, having lost their entire hydrogen envelope at the top of the asymptotic giant branch. They then evolve very rapidly, on a timescale of $10^{3}-10^{4} \mathrm{yr}$, at a constant luminosity of $10^{4} \mathrm{~L}_{\odot}$ and eventually become DB white dwarfs.

Landolt (1975) discovered that HD 160641 was variable with a most likely period of $\sim 0.6 \mathrm{~d}$. This result was supported by subsequent observations by Walker \& Kilkenny (1980). Simultaneous photometric and spectroscopic obser- 

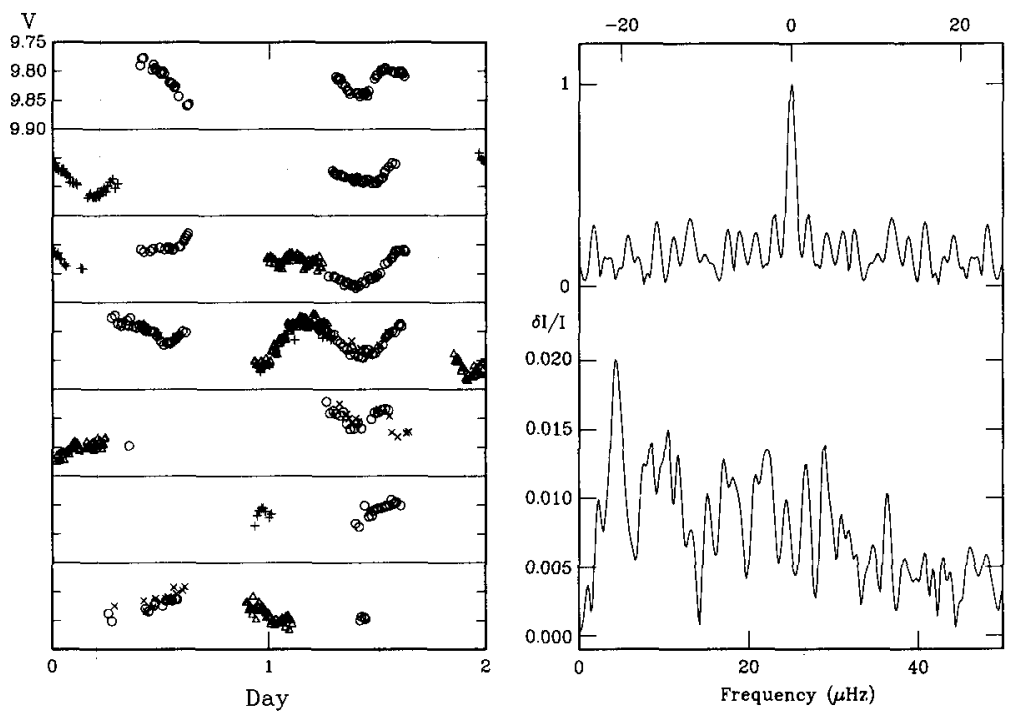

Figure 1. Left: $V$ photometry for the 'core' campaign (2000 May 30 - June 12). The panels run continuously from left to right and top to bottom, covering HJD $2451696-2451710$, with no repeated data. Symbols are: MJUO $(\triangle)$, SSO $(+)$, SAAO $0.5-\mathrm{m}(\circ)$ and SAAO $0.75-\mathrm{m}$ Alan Cousins Telescope $(x)$. Right: The spectral window (upper line) normalised to unity at peak and the amplitude periodogram (lower line) for the 'core' campaign, where the ordinate units are fractional intensity, $\delta \mathrm{I} / \mathrm{I}$.

vations by Lynas-Gray et al. (1987) showed HD 160641 to have four, or perhaps five, periods and to have a mass of $\sim 1 \mathrm{M}_{\odot}$ which is a little higher than might be expected from Schönberner's (1977) evolutionary tracks. Observations reported here in preliminary form are intended to verify the analysis of Lynas-Gray et al. (1987) and to check for period changes expected on a time scale of a decade or so, given the rapid evolution which Schönberner's (1977) calculations suggest. They were also an improvement on the earlier data because they were made from three sites distributed in longitude to permit (in principle at least) continuous monitoring for an interval of $18 \mathrm{hr}$.

\section{Photometry}

During the 'core' campaign in 2000, photometry (Fig. 1, left) was obtained at the South African Astronomical Observatory (SAAO), Siding Springs Observatory (SSO) in Australia and at Mount John University Observatory (MJUO) in New Zealand. The photometry from this campaign produced a very clear window function but a rather noisy periodogram (Fig. 1, right). Some of the periods extracted from the data were not consistent with the prior photometric study carried out by Lynas-Gray et al. (1987). The periods extracted from the 2000 
Table 1. The three highest amplitude frequencies extracted from the 1982 data (Lynas-Gray et al. 1987), the 'core' 2000 campaign, and the forced fit to the 1982 data assuming aliasing.

\begin{tabular}{rcrcrc}
\hline \multicolumn{2}{c}{1982} & \multicolumn{2}{c}{2000 'core' } & \multicolumn{2}{c}{1982 'forced' } \\
$\begin{array}{c}\text { Freq } \\
(\mu \mathrm{Hz})\end{array}$ & $\begin{array}{c}\text { Amp } \\
(\mathrm{mag})\end{array}$ & $\begin{array}{c}\text { Freq } \\
(\mu \mathrm{Hz})\end{array}$ & $\begin{array}{c}\text { Amp } \\
(\mathrm{mag})\end{array}$ & $\begin{array}{c}\text { Freq } \\
(\mu \mathrm{Hz})\end{array}$ & $\begin{array}{c}\text { Amp } \\
(\mathrm{mag})\end{array}$ \\
\hline 16.29 & 0.029 & 4.21 & 0.020 & 16.31 & 0.026 \\
10.48 & 0.021 & 22.28 & 0.012 & 22.05 & 0.016 \\
6.69 & 0.017 & 29.04 & 0.012 & 6.68 & 0.020 \\
\hline \hline
\end{tabular}

campaign and the 1982 study are shown in Table 1 . Also shown is the frequencies from the 1982 study when the two close frequencies are forced to the 2000 values (labelled ' 1982 forced'). There are two frequencies that are considered likely: the $22.28 \mathrm{mHz}(0.52 \mathrm{~d})$ and the $4.21 \mathrm{mHz}(2.75 \mathrm{~d})$. There is the possibility that the $10.48 \mathrm{mHz}$ frequency is a $1-\mathrm{d}$ alias of the $22.28 \mathrm{mHz}$ one.

\section{Spectroscopy}

Stacked plots were used to investigate the line profile variations and radial velocities were determined for most observed lines. In Fig. 2 the data from the MJUO 1-m telescope and HERCULES spectrograph (Hearnshaw et al. 2002) on 2003 June 17 clearly shows the variation of an unidentified feature alongside the He I $5875 \AA$ line and possible variation of the HeI $5875 \AA$ line itself. The HeII $6560 \AA$ line does not appear to demonstrate any clear variation.

The velocities obtained from the 2000 data indicate differences between line species and ionisation levels. All the lines observed, except the CIII $5696 \AA$ emission line, varied in phase with amplitudes of up to $\sim 25 \mathrm{~km} \mathrm{~s}^{-1}$. The CIII line showed little velocity variation and had the lowest velocity observed. This suggests that the CIII emission line originates in the stellar wind rather than in the photosphere.

A detailed analysis of the more recent data, and another spectroscopic campaign with the aim of high signal-to-noise data, is required for a complete understanding of this star's spectral variation.

\section{References}

Aller, L.H. 1954, Mem. Soc. R. Sci. Leige 14, 353

Bidelman, W.P. 1952, ApJ, 116, 227

Hamman, W.R. et al. 1982, A\&A, 116, 273

Hearnshaw, J.B. et al. 2002, Experimental Astronomy, 13, 59

Landolt, A.U. 1975, ApJ, 196, 789

Lynas-Gray, A.E. et al. 1987, MNRAS, 227, 1073

Rauch, T. 1996, in ASP Conf. Ser. Vol. 96, Hydrogen Deficient Stars, eds C.S. Jeffery \& U. Heber, (ASP: San Francisco), 174 

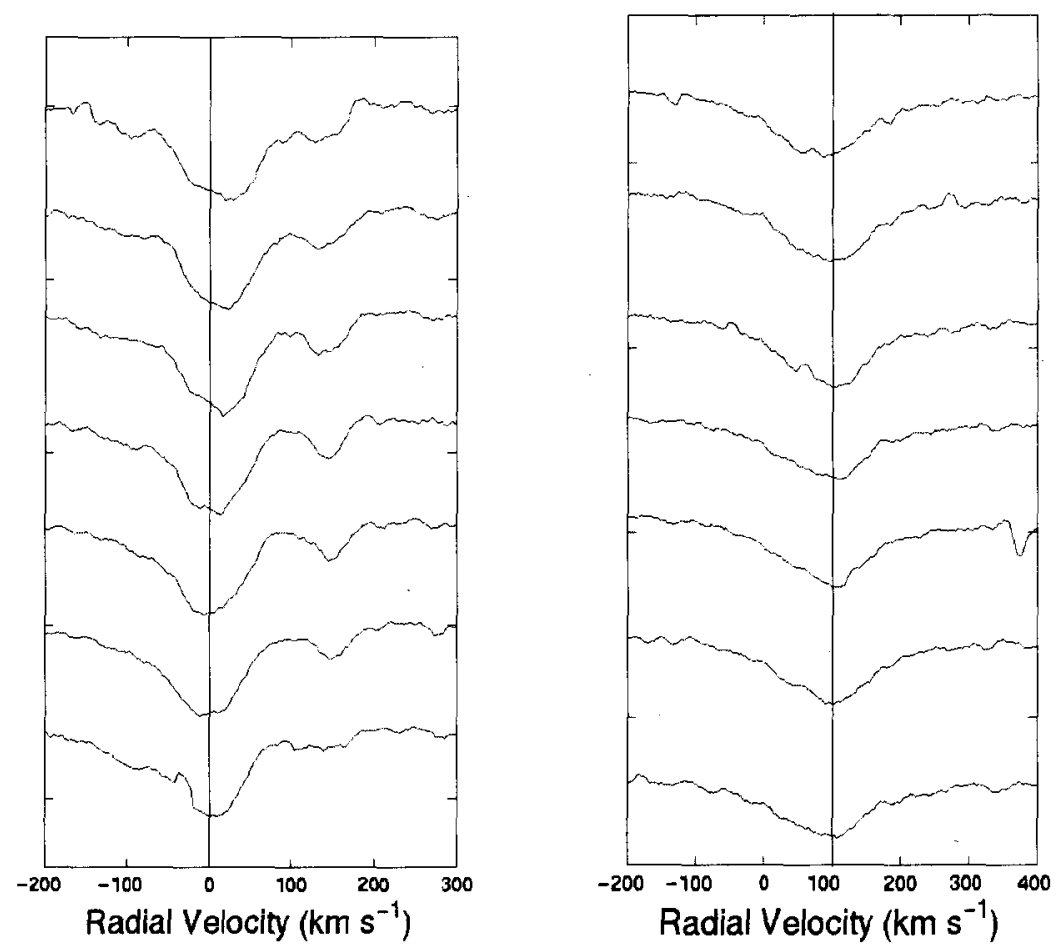

Figure 2. High signal-to-noise spectra taken with the HERCULES spectrograph at MJUO on 2003 June 17 over a 5.5-hr interval, plotted in velocity space. Left panel: The HeI $5875 \AA$ triplet. Right panel: The HeII $6560 \AA$ line.

Schönberner, D., Wolf, R.E.A. 1974, A\&A, 37, 87

Schönberner, D. 1977, A\&A, 57, 169

Walker, H.J., Kilkenny, D. 1980, MNRAS, 190, 299 